\title{
Lung function and bronchodilator response in 4-year-old children with different wheezing phenotypes
}

\author{
E. Oostveen*, S. Dom”, K. Desager", M. Hagendorens`, W. De Backer* and J. Weyler"
}

ABSTRACT: Persistent wheeze is a common chronic disease in early childhood and later may progress to asthma. However, the association between pre- and post-bronchodilator lung function and the wheezing phenotype in preschool children is not known.

Children 4 yrs of age involved in a prospective birth cohort study (in Antwerp, Belgium) concerning perinatal factors and the occurrence of asthma and allergies, were invited to participate in lung function measurements with the forced oscillation technique. The wheezing phenotype was assessed via (bi)annual questionnaires.

Wheezing phenotype and baseline respiratory impedance data were available for 325 children, $96 \%$ of whom underwent bronchodilation tests. The baseline resistance at $4 \mathrm{~Hz}$ was higher in children with early transient $\left(11.0 \mathrm{hPa} \cdot \mathrm{s} \cdot \mathrm{L}^{-1}, \mathrm{n}=127\right)$ or persistent wheeze $\left(11.9 \mathrm{hPa} \cdot \mathrm{s} \cdot \mathrm{L}^{-1}, \mathrm{n}=54\right)$ than in children who never wheezed $\left(10.3 \mathrm{hPa} \cdot \mathrm{s} \cdot \mathrm{L}^{-1}, \mathrm{n}=144\right)$. After bronchodilation, the resistance decreased on average by $22 \%$. The decrease was greater among the persistent wheezers than among those who never wheezed (3.4 versus $\left.2.3 \mathrm{hPa} \cdot \mathrm{s} \cdot \mathrm{L}^{-1}\right)$.

The baseline lung function was poorer and the bronchodilator response was greater in 4-yr-old children with persistent wheeze than in those who never wheeze or who had early transient wheeze, implying a higher bronchomotor tone in the former group.

KEYWORDS: Childhood asthma, cohort studies, forced oscillations, preschool children, wheezing ersistent wheezing is a common chronic disease in young children and a major cause of preschool morbidity. The prevalence of recurrent days with cough, wheeze and/or breathlessness in children aged 1-5 yrs has been reported to be $32 \%$, and as many as $24 \%$ of preschool children suffer from weekly symptoms despite current treatment [1].

Atopy and increased airway responsiveness in young children have been associated with persistent wheeze and asthma in later life [2-4]. Furthermore, abnormalities in pulmonary function during infancy have been demonstrated to be an important determinant of subsequent respiratory symptoms and lung function, independently of atopy and airway responsiveness [5-7]. It was recently reported that persistent wheeze and low airway function at school age are independently associated with chronic asthma in early adulthood [8]. These findings have increased our awareness of the impact of respiratory events in early life on the development of respiratory disease in adulthood.
At present, however, children at high risk of developing persistent asthma are still inadequately identified [9].

Recent advances in diagnostic technologies have led to the standardisation of a number of techniques for the assessment of lung function in the preschool age group [10]. The use of these techniques in preschool children with recurrent wheeze may have important implications for the correct identification of children at risk of developing severe asthma in later life. BRUSSEE et al. [11] recently reported that 4-yr-old children with persistent wheeze exhibited higher interruptor resistances than age-matched children who never wheezed or those with the early transient wheezing phenotype.

Asthma is at least partially defined by abnormalities in pulmonary function, including variable airway obstruction. In addition to reporting on baseline lung function measures (respiratory resistance and reactance, as determined with the forced
AFFILIATIONS

*Dept of Pulmonary Medicine, "Dept of Paediatrics, Antwerp University Hospital and University of Antwerp, and

${ }^{\#}$ Dept of Epidemiology and Social Medicine, University of Antwerp, Antwerp, Belgium.

CORRESPONDENCE

E. Oostveen

Dept of Pulmonary Medicine Antwerp University Hospital Wilrijkstraat 10 B-2650 Edegem-Antwerp Belgium

E-mail: Ellie.0ostveen@uza.be

Received:

Feb 112009

Accepted after revision:

Nov 042009

First published online:

Nov 192009 
oscillation technique) and the bronchodilator responsiveness in 4yr-old children, the present study also aimed to determine the association between baseline and post-bronchodilator resistance and reactance, and the phenotypes of childhood wheezing.

\section{METHODS}

\section{Study design and study population}

The Prospective Cohort on the Influence of Perinatal Factors on the Occurrence of Asthma and Allergies (PIPO) study is a prospective birth cohort study involving 1,128 children. Between June 1997 and December 2001, pregnant females in the Antwerp region of Belgium were invited to participate by completing a screening questionnaire. Data on demography, respiratory symptoms and risk factors for asthma were collected through the use of postal questionnaires: the first questionnaire covered the first year of life, with subsequent questionnaires at 6-month intervals up to the age of 4 yrs. The questionnaires were based on validated International Study of Asthma and Allergies in Childhood (ISAAC) questionnaires [12]. At 4 yrs of age, the children included in the PIPO study were invited for medical examination and lung function testing at the Antwerp University Hospital (Antwerp, Belgium). The children who regularly used bronchodilators were asked to abstain from their use on the examination day, whereas the use of inhaled corticosteroids was not interrupted. The atopic status of the child and parents was assessed from specific immunoglobulin (Ig)E levels against common food and inhalant allergens, determined from blood samples. All parts of the study were approved by the University Ethics Committee (University of Antwerp, Antwerp, Belgium), and written informed parental consent was obtained before each assessment.

\section{Lung function measurements}

\section{Forced oscillation technique}

Respiratory impedance ( $\mathrm{Zrs}$ ) was measured in the frequency range $4-32 \mathrm{~Hz}$, using a home-made setup that met the American Thoracic Society (ATS)/European Respiratory Society (ERS) requirements [10, 13]. Measurements were performed according to recent international guidelines [10]. Recordings lasted for $16 \mathrm{~s}$, and the average of three to five acceptable Zrs data was used for further analysis.

\section{Measurement protocol}

Children sitting upright on their parent's lap were measured with their head in a neutral position. The parent supported the child's chin and cheeks with both hands. The child was instructed to breathe normally while watching a video film.

\section{Reversibility testing}

After the baseline Zrs measurement, $200 \mu \mathrm{g}$ of salbutamol was administered through a spacer (OptiChamber; Respironics New Jersey Inc., Parsipanny, NJ, USA) without an additional face mask or a mouthpiece. The Zrs measurement was repeated $15 \mathrm{~min}$ thereafter. The pre- and post-bronchodilator measurements were performed by the same technician.

\section{Short-term reproducibility}

In 50 children, chosen at random, the short-term reproducibility of the Zrs data was assessed by repeating the baseline measurement after a 15-min time interval.

\section{Wheezing phenotype}

Symptoms of wheeze were assessed through core questions from the ISAAC questionnaire [12]. According to the history of wheeze reported by the parents in the (bi)annual questionnaires, the children were divided into those who never wheeze, and those with early transient, late-onset or persistent wheeze $[2,11]$.

\section{Data analysis}

From the measured respiratory reactance (Xrs) data, the "area under the reactance curve" (AX), i.e. the integrated area of all $X$ rs data below zero up to the resonance frequency (fres), was determined. Data are expressed as mean $\pm \mathrm{SD}$, unless otherwise specified. Statistical analyses were performed using SPSS for Windows, Version 15.0 (SPSS Inc., Chicago, IL, USA).

After testing the data for normality, the difference between the two baseline measurements was analysed with paired t-tests. The differences between the four groups of wheezing phenotype were assessed with one-way ANOVA, post hoc least significant difference analysis or Mann-Whitney analysis (or Chi-squared test). Significance was accepted at $\mathrm{p}<0.05$.

The online supplementary material provides additional details on the measurement technique, the questionnaires, the definitions of the wheezing phenotypes and the analysis of adjustment for potential confounders.

\section{RESULTS}

Baseline Zrs data were obtained for 535 children and postbronchodilator Zrs data for 501 children (the online supplementary material includes a flowchart of the children participating in the study). In 203 children (38\%), the wheezing phenotype could not be determined because of incomplete or missing questionnaires. These children were excluded from the study group. The remaining children $(n=332)$ were divided into those who never wheeze $(n=144,43 \%)$, and those with an early transient $(n=127,38 \%)$, a late-onset $(n=7,2 \%)$ or a persistent $(n=54,16 \%)$ wheezing phenotype. The group of children with late-onset wheeze was considered too small for further analysis. In 313 of the remaining 325 children (96\%), the post-bronchodilator response was also assessed. The general characteristics of the subgroups are listed in table 1. The anthropometric characteristics and sex distribution were not different in the children with the different wheezing phenotypes. In comparison with the group who never wheeze, a significantly higher proportion of the persistently wheezing children were atopic and had used antibiotics at 4 yrs of age, whereas the children with early transient or persistent wheeze suffered from lower respiratory tract infections at 4 yrs of age significantly more frequently.

The short-term reproducibility of Zrs was assessed in 27 young males and 23 young females. This group of children did not differ significantly from the study group ( $n=325$ children) in terms of the distribution of the wheezing phenotypes (in 12 children, the wheeze phenotype was undetermined; 18 children had never wheezed; 16 children had early transient wheeze phenotype; four children had a persistent wheeze phenotype), or the value of $R 4$ (resistance at $4 \mathrm{~Hz}$ ) or $\mathrm{AX}$ (see later). The results of the two baseline measurements of respiratory resistance $(R r s)$ and $X r s$ are depicted in figure 1. The Rrs data 


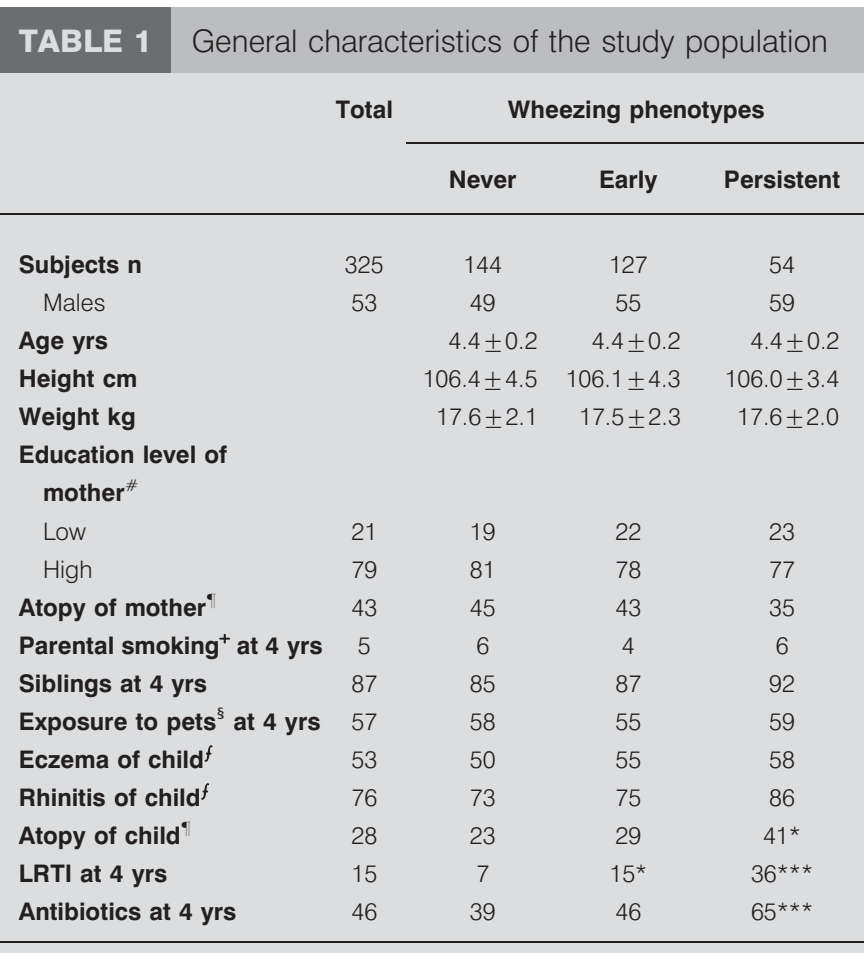

Data are presented as mean \pm SD or $\%$, unless stated otherwise. LRTI: lower respiratory tract infection (a serious lung or airways infection). ${ }^{*}$ : high if the mother had completed a bachelor or master's degree, otherwise low; ": defined as a specific immunoglobulin $E$ level $\geqslant 0.35 \mathrm{kU} \cdot \mathrm{L}^{-1}$ at the medical examination at 1 or $4 \mathrm{yrs}^{+}{ }^{+}$: regular exposure to tobacco smoke by either of the parents; s: regular exposure to a cat or dog; ${ }^{f}$ : a positive answer to the following question in any of the questionnaires: "Did your child suffer from eczema/rhinitis during the past 6 months?" [12]. At 4 yrs: a positive answer in the questionnaire returned at 42 or 48 months. ${ }^{*}: p<0.05$, relative to those who never wheeze; $* * *: p<0.001$, relative to those who never wheeze.

obtained 15 min apart were not significantly different, except that $R_{6}$ and $R 8$ were slightly lower (by $\sim 0.4 \mathrm{hPa} \cdot \mathrm{s} \cdot \mathrm{L}^{-1}$ ) in the second baseline measurement $(\mathrm{p}<0.05)$. A small upward shift was noted in the second baseline $X$ rs curve relative to the first at almost all data points $(\mathrm{p}<0.01)$; the $\mathrm{Xrs}$ data from the second baseline measurement were on average $0.4 \mathrm{hPa} \cdot \mathrm{s} \cdot \mathrm{L}^{-1}$ larger than those from the first measurement.

The baseline $R r s$ and $X r s$ in the groups of children with different wheezing phenotypes are reported in figure 2. Rrs displayed a slight negative frequency dependence in the children who never wheeze. Rrs increased in magnitude and the negative frequency dependence of Rrs became progressively more marked in the groups of children with the early transient or persistent wheezing phenotypes. In comparison with the children who never wheeze, Xrs became more negative and exhibited an ever-higher fres in the sequence early transient and persistent wheezing phenotype (see table 1 of the online supplementary material). Since the largest differences in $\mathrm{Zrs}$ at baseline between the wheezing phenotype groups were observed in the low-frequency range, our subsequent analysis was focused on those frequencies. R4, $R 6, R 8$, reactance at $4 \mathrm{~Hz}\left(X_{4}\right)$, and $X_{6}, X_{8}$, and $\mathrm{AX}$ were analysed. Figure 3 presents the values of $R 4$ and $A X$ at baseline

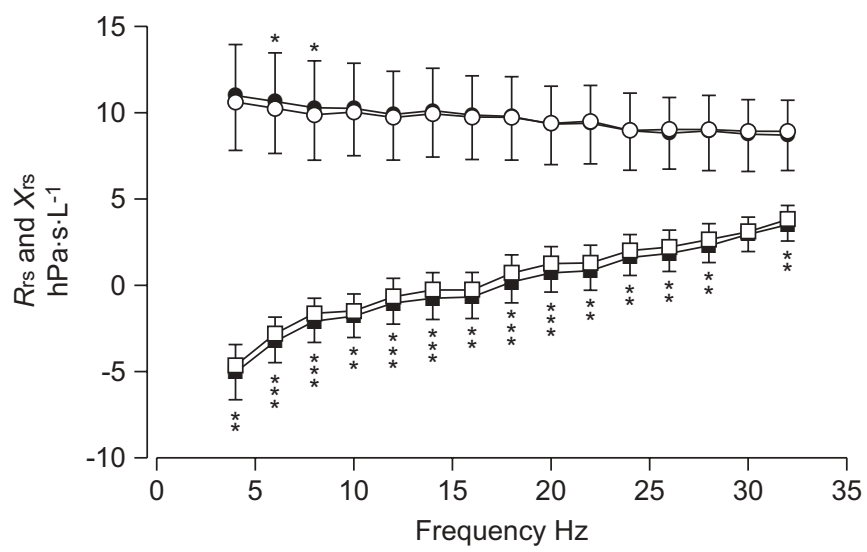

FIGURE 1. Short-term reproducibility of respiratory resistance (Rrs; 0 and 0 ) and respiratory reactance ( $X_{\mathrm{rs}} ; \mathbf{\square}$ and $\square$ ) data obtained from 50 4-yr-old children The two baseline measurements ( $\square$ and $\mathbf{0}$ : baseline $1 ; \square$ and $\bigcirc$ : baseline 2) were made 15 min apart. Data points and error bars present average values \pm SD. *: $p<0.05 ; * *: p<0.01 ; * * *: p<0.001$

and after bronchodilation. The children with early transient, late-onset or persistent wheeze had baseline values of $R 4$ and AX that were significantly larger than those of the children who never wheeze. Further, the children with persistent wheeze had significantly larger baseline $R 4$ values than the children with early transient wheeze.

Bronchodilation significantly decreased the values of $R 4$ and $\mathrm{AX}$ in all the groups of children. On average, $R 4$ decreased $22 \%$ by bronchodilation. However, for the children with persistent wheeze, the decrease in $R 4$ after bronchodilation was significantly larger than the children who never wheeze and those with early transient wheeze $(\mathrm{p}<0.05)$. After bronchodilation, the values of $R 4$ were still significantly larger in the children with persistent wheeze than in those who never wheeze $(p<0.02)$. Relative to the group who never wheeze, the decreases in AX were significantly larger in the children with early transient and persistent wheeze, and accordingly the post-bronchodilator values of $\mathrm{AX}$ were comparable in the three groups of children with the different wheezing phenotypes (fig. 3 and table 2).

The characteristics of the children who never wheeze were used to determine the cut-off values for a significant bronchodilator response. From the fifth percentiles, the cutoff points were set to be absolute decreases of $5.5 \mathrm{hPa} \cdot \mathrm{s} \cdot \mathrm{L}^{-1}$ and $31.0 \mathrm{hPa} \cdot \mathrm{L}^{-1}$ for $\mathrm{R} 4$ and $\mathrm{AX}$, respectively, and relative decreases of $43 \%$ and $81 \%$, respectively. Compared with the relative change, expressing the bronchodilator response in absolute change proved to be a more sensitive means of differentiating between the groups (table 3). Significantly more children with a persistent wheeze $(13 \%)$ than children who never wheeze $(4 \%)$ responded positively to bronchodilation when the absolute change in $R 4$ was considered $(p<0.05)$. When the absolute change in AX was used to define a positive response, significantly more children with an early transient $(14 \%)$ or persistent wheeze $(23 \%)$ responded significantly to bronchodilation than the children who never wheeze $(4 \%)$. The combined changes in resistance and reactance at $4 \mathrm{~Hz}$ after 

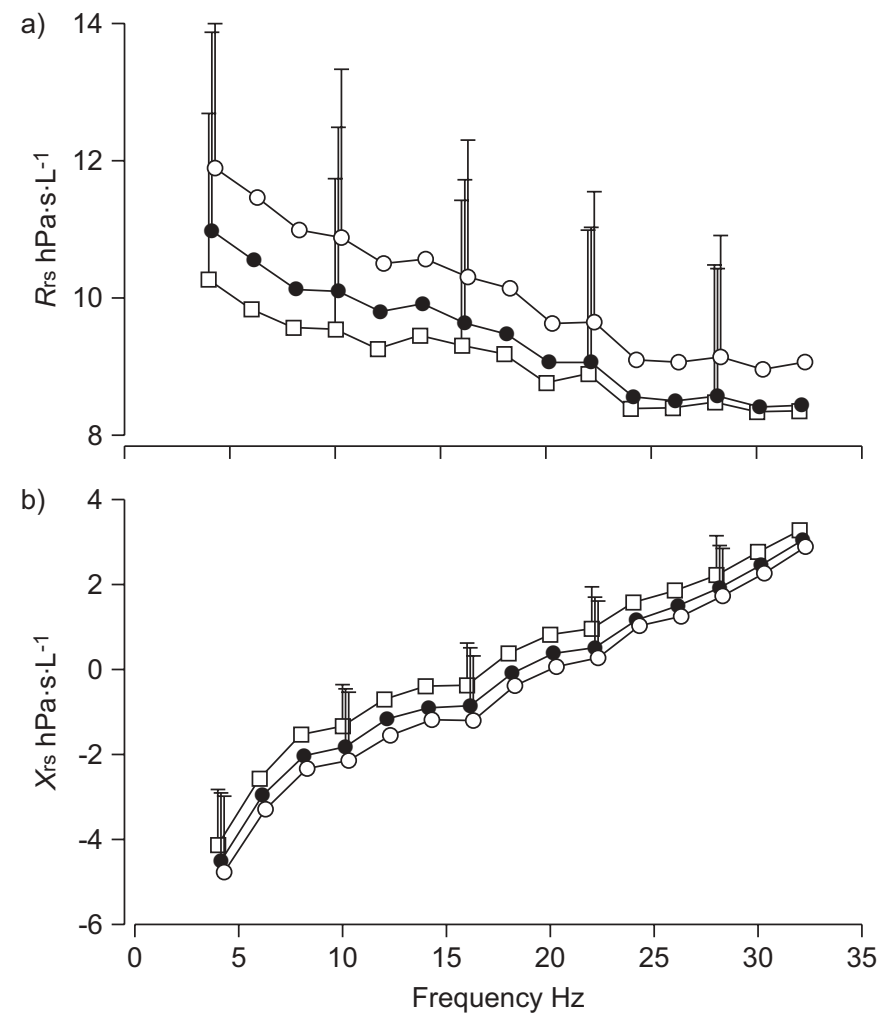

FIGURE 2. Average $\pm S D$ of a) respiratory resistance (Rrs) and b) respiratory reactance $\left(X_{r s}\right)$ in the groups of children with different wheezing phenotypes: those who never wheeze $(\square ; n=144)$, those with early transient wheeze $(\bullet ; n=127)$ and those with persistent wheeze $(O ; n=54)$.

bronchodilation in the different groups of wheeze phenotype followed the same path (fig. 4).

\section{DISCUSSION}

Although there was a considerable overlap in lung function data between the groups, our study did reveal significant differences both in the baseline respiratory function and in the bronchodilator response between preschool children with different wheezing phenotypes. At 4 yrs of age, the children with early transient wheeze yielded higher baseline resistance values than those who never wheezed, although $43 \%$ of the children with early transient wheeze had experienced only one wheezing episode during the first 3 yrs of life. The children with persistent wheeze, of whom two-thirds had experienced several episodes of wheeze during their fourth year of life, displayed a poorer baseline lung function than the children who never wheeze or those with early transient wheeze, and their bronchodilator response was larger.

There was a considerable dropout rate in our study: half of the children who entered the cohort at birth were not available for examination at $4 \mathrm{yrs}$ of age and $38 \%$ of the remaining had incomplete or missing questionnaires. However, despite the loss of data, we feel that the dropout does not affect or limit the interpretation of our results. We did not intend to study lung function and wheeze phenotype in a representative sample of the population; the purpose of our study was rather to investigate the association between lung function and wheezing
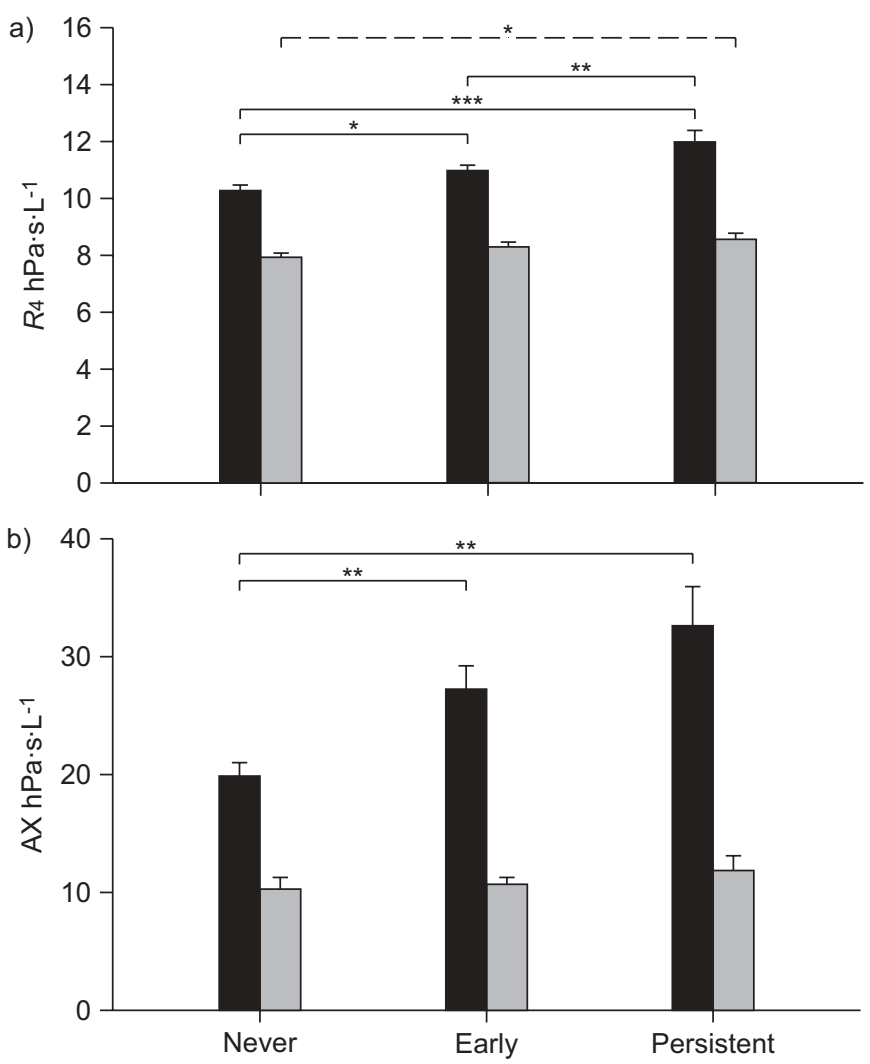

FIGURE 3. Baseline ( $\mathbf{\square})$ and post-bronchodilator ( $\square$ ) values of a) resistance at $4 \mathrm{~Hz}(R 4)$ and b) area under the reactance curve (AX) in the groups who never wheeze, or who have early transient or persistent wheeze. Bars and error bars present average values \pm SEM. *: $p<0.05 ; * *: p<0.01 ; * * *: p<0.01$. _-: comparisons between baseline values; - - -: comparison between postbronchodilator values.

phenotype. From the description of our study population (table 1), it is clear that the mothers of the children were relatively highly educated (which may explain the low smoking incidence) and atopic mothers were more likely to enrol their child into our birth cohort. We do not consider this nonrepresentative selection at entry as a drawback; on the contrary, it favoured a more homogeneous distribution of the children among the wheeze phenotype groups.

Following the Tucson definition [2], the ERS Task Force on wheezing disorders in preschool children recently described cut-off ages to define the wheeze phenotype of the child at 3 and 6 yrs of age [14], whereas we defined cut-off points at 3 and 4 yrs of age. Many previous epidemiological studies, however, employed different definitions of the wheeze phenotype: cut-off ages of 3 and 5 yrs [15, 16], 3 and 7 yrs [17], and 4 and 10 yrs [18] have been described. Our definition of wheeze phenotype was similar to that employed by BRUSSEE et al. [11].

The baseline reproducibility of $R$ rs indicated isolated, small decreases $(\sim 3 \%)$ in $R 6$ and $R 8$ and a significant upward shift of the $X r$ curve throughout the whole frequency range at the second baseline measurement compared with the first (fig. 1). The most probable explanation appears to be a change in chest wall compliance due to a more relaxed state in the young 
TABLE 2 Baseline resistance and reactance values and the change after bronchodilation in the groups of children with different wheezing phenotypes

\begin{tabular}{|c|c|c|c|}
\hline & Never & Early & Persistent \\
\hline \multicolumn{4}{|l|}{ Baseline } \\
\hline Subjects $n$ & 144 & 127 & 54 \\
\hline$R 6 \mathrm{hPa} \cdot \mathrm{s} \cdot \mathrm{L}^{-1}$ & $9.8(9.5-10.2)$ & $10.5(10.1-11.0)^{\star}$ & $11.5(10.7-12.2)^{\star \star *, \# \#}$ \\
\hline$R 8 \mathrm{hPa} \cdot \mathrm{s} \cdot \mathrm{L}^{-1}$ & $9.6(9.2-9.9)$ & $10.1(9.7-10.6)$ & $11.0(10.3-11.7)^{\star \star \star \star \#}$ \\
\hline$X_{8} \mathrm{hPa} \cdot \mathrm{s} \cdot \mathrm{L}^{-1}$ & $-1.5(-1.7--1.4)$ & $-2.0(-2.3--1.8)^{\star *}$ & $-2.3(-2.8--1.9)^{\star *}$ \\
\hline $\mathrm{AX} h \mathrm{ha} \cdot \mathrm{L}^{-1}$ & $19.9(17.6-22.1)$ & $27.2(23.4-31.1)^{\star *}$ & $32.6(25.8-39.3)^{\star \star \star}$ \\
\hline \multicolumn{4}{|l|}{ Bronchodilation } \\
\hline Subjects $n$ & 139 & 121 & 53 \\
\hline$\Delta R 4 \mathrm{hPa} \cdot \mathrm{s} \cdot \mathrm{L}^{-1}$ & $-2.3(-2.6--2.0)$ & $-2.6(-3.1--2.2)$ & $-3.4(-4.1--2.7)^{\star . \#}$ \\
\hline$\Delta R 6 \mathrm{hPa} \cdot \mathrm{s} \cdot \mathrm{L}^{-1}$ & $-2.1(-2.4--1.9)$ & $-2.5(-2.9--2.2)$ & $-3.2(-3.8--2.5)^{\star *}$ \\
\hline$\Delta \mathrm{AX} \mathrm{hPa} \cdot \mathrm{L}^{-1}$ & $-9.8(-11.6--8.1)$ & $-16.3(-19.6--13.0)^{\star \star}$ & $-21.2(-27.4--15.1)^{\star \star \star}$ \\
\hline
\end{tabular}

Data are presented as mean $(95 \% \mathrm{Cl})$, unless stated otherwise. $R 4, R 6, R 8$ and $\mathrm{X}_{4}, \mathrm{X}_{6}, \mathrm{X}_{8}$ : resistance and reactance, respectively, at 4,6 and $8 \mathrm{~Hz}$; $\mathrm{AX}$ : area under the reactance curve; $\Delta$ : change after bronchodilation. ${ }^{*}: p<0.05$, relative to the children who never wheeze; ${ }^{*}: p<0.01$, relative to the children who never wheeze; $* * *$ : $p<0.001$, relative to the children who never wheeze; ${ }^{\#}: p<0.05$, relative to the children with early transient wheeze; ${ }^{\# \#}: p<0.01$, relative to the children with early transient wheeze.

children, who had adapted somewhat to the new experimental situation. Although there was a systematic difference in Xrs between the two baseline measurements, the change was small relative to that induced by bronchodilation: the relative increases in $X_{4}$ and $X_{6}$ were $6 \%$ and $12 \%$, respectively, whereas the changes induced by bronchodilation were $24 \%$ and $35 \%$, respectively.

Besides the analysis of the raw Rrs and Xrs data points at low frequency, we also analysed AX, all Xrs data points below fres thus being taken into account. As such, AX can be regarded as an index reflecting the elastance of the respiratory system, and it actually has the dimension of elastance. Its recent introduction was because $\mathrm{AX}$ is not greatly influenced by the measurement noise of the individual Xrs data points [19]. Indeed, AX discriminated between the groups with different wheezing phenotypes better or with more power (table 2 ) than $X_{4}, X_{6}$ or $X_{8}$.

In contrast with most previous forced oscillation technique (FOT) measurements in children, we obtained highly reproducible $\mathrm{Zrs}$ data at $4 \mathrm{~Hz}$ (see the online supplementary material), and hence we chose to analyse $R$ 4. It should be noted, however, that similar results were obtained when R6 and $R 8$ were considered (table 2). Our results are in close agreement with those of BRUSSEE et al. [11], who prospectively investigated the interrupter airway resistance (Rint) in children 4 yrs of age; they employed similarly defined wheezing phenotypes, but included 2.5 times more children in their study than we did. They found that children with persistent wheeze had a higher baseline Rint than those who never wheezed. In contrast, our FOT resistance also differentiated the group of children with early transient wheeze from the group of children who never wheeze, and the group of children with persistent wheeze from the group of children with early transient wheeze. This suggests that resistance measurements with FOT are more sensitive than Rint measurements in children of this age. In accordance with this, DELACOURT et al. [20] concluded that FOT is more sensitive than Rint measurements for the detection of obstruction and its reversibility in children with asthma or a chronic cough.

\begin{tabular}{|c|c|c|c|c|}
\hline \multirow[t]{2}{*}{ TABLE 3} & \multicolumn{4}{|c|}{$\begin{array}{l}\text { Number of children in each group giving a } \\
\text { significant bronchodilator response as } \\
\text { determined by the fifth percentile for the children } \\
\text { who never wheeze }\end{array}$} \\
\hline & & Never & Early & Persistent \\
\hline \multicolumn{2}{|l|}{ Subjects n } & 139 & 121 & 53 \\
\hline \multicolumn{2}{|c|}{$|\Delta R 4|>5.5 \mathrm{hPa} \cdot \mathrm{s} \cdot \mathrm{L}^{-1}$} & $6(4)$ & $6(5)$ & 7 (13) \\
\hline \multicolumn{2}{|c|}{$|\Delta \mathrm{AX}|>31.0 \mathrm{hPa} \cdot \mathrm{L}^{-1}$} & $6(4)$ & $17(14)$ & $12(23)$ \\
\hline \multicolumn{2}{|c|}{$|\Delta R 4 / R 4|>43 \%$} & $7(5)$ & $6(5)$ & $5(9)$ \\
\hline \multicolumn{2}{|c|}{$|\triangle \mathrm{AX} / \mathrm{AX}|>81 \%$} & $6(4)$ & $9(7)$ & 8 (15) \\
\hline
\end{tabular}

Data are presented as $n(\%)$, unless otherwise indicated. Values are presented as modulus. Numbers in bold indicate a significant difference relative to the group of children who never wheeze ( $p<0.05$, Pearson's Chi-squared test). R4: resistance at $4 \mathrm{~Hz}$; $\mathrm{AX}$ : area under the reactance curve. 


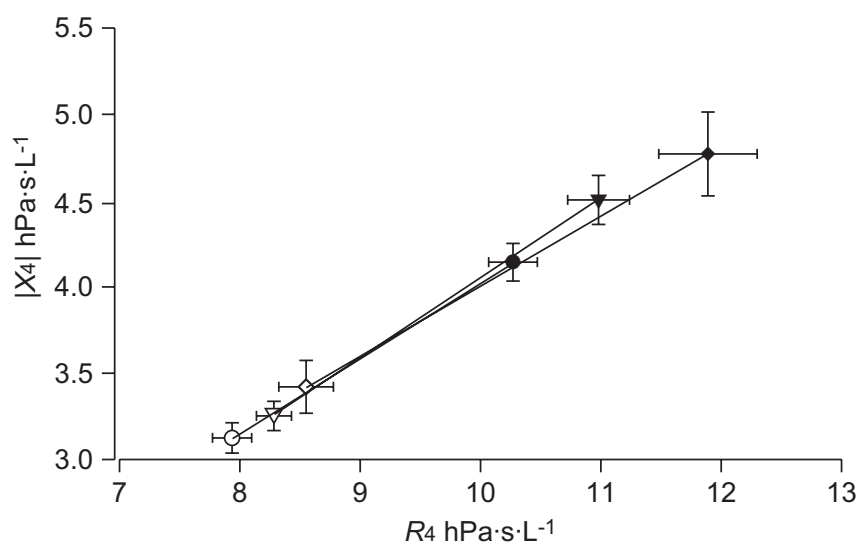

FIGURE 4. Relationship between baseline (closed symbols) and postbronchodilator values (open symbols) of reactance at $4 \mathrm{~Hz}\left(X_{4}\right)$ and resistance at $4 \mathrm{~Hz}(R 4)$ in the groups of children who never wheeze $(\bullet)$, or who have early transient wheeze $(\boldsymbol{\nabla})$ or persistent wheeze $(\bullet)$. Average values \pm SEM are shown.

LOWE et al. [21] studied the specific airway resistance (sRaw) in 3-yr-old children taking part in a prospective birth cohort. sRaw was found to be significantly higher in the children who had experienced more than two wheezing episodes than in the children who had never wheezed. In our study, the children who had stopped wheezing by 3 yrs of age (the children with early transient wheeze) still had a significantly higher respiratory resistance at 4 yrs of age than the children who had never wheezed at all.

As far as we are aware, the bronchodilator response has not been assessed previously in preschool children with different wheezing phenotypes. We detected significant differences in the responses to a short-acting bronchodilator: the children with persistent wheeze displayed a larger decrease in Rrs than those children who never wheeze or who have early transient wheeze (table 2). It is noteworthy that the baseline $X_{\mathrm{rs}}$ values and the change after bronchodilator administration also distinguished the different wheezing phenotype groups. In contrast with Rrs at low frequency, the post-bronchodilator Xrs data were all very similar among the groups with different wheezing phenotypes. How should these data to be interpreted?

A higher baseline Rrs was always associated with a lower Xrs at low frequency, and thus with higher values of $\mathrm{AX}$ and fres (table 2 and table 1 of the online supplementary material). Further, the effect of the short-acting bronchodilator was an upward shift in Xrs, which was largest in the groups with the lowest baseline $X$ rs values. There are a number of mechanisms that may explain the concomitant changes in Rrs and Xrs. Perhaps the most probable explanation for lower $X$ rs values in small children with larger values of airway resistance is the effect of the upper airway shunt [22]. This shunt effect, exerted by the elastic properties of the soft tissues of the cheeks and floor of the mouth, increases as the airway resistance is increased. It results in a phase shift between pressure and flow, especially at low frequency, with the net effect that Xrs decreases as the airway resistance increases. A similar effect is obtained when the peripheral airway constriction in these young children is sufficiently severe and inhomogeneous to cause peripheral time constant differences and an altered frequency dependence of the pulmonary impedance [23]. This mechanism was earlier offered as the most likely explanation for the increase in apparent tissue compliance observed after bronchodilation in adult asthmatics [24]. Both of the previously mentioned mechanisms explain the parallel changes observed in Rrs and Xrs after bronchodilation. Indeed, all the wheezing phenotype groups exhibited a joint path of bronchodilator-induced changes in $R 4$ and $X_{4}$ (fig. 4). Children with more constricted airways display higher resistances associated with more negative reactances. The bronchodilator-induced changes in both resistance and reactance are larger in these children, which results in a rather uniform post-bronchodilator impedance among the groups.

The bronchodilator responsiveness in our groups of 4-yr-old children was remarkably large. In the children who never wheeze, an average decrease of $22 \%$ in $R 4$ was observed after bronchodilation, indicating a significant bronchomotor tone at baseline. When we used the coefficient of repeatability of $R 4$ [10] as a cut-off value, as many as $35 \%$ of the children who never wheezed demonstrated a significant positive bronchodilator response. To the best of our knowledge, the bronchodilator responsiveness of children who never wheeze has not been assessed previously in a prospective study. In crosssectional studies, however, the bronchodilator response of healthy young children has been reported to be significant. THAMRIN et al. [25] and MALMBERG et al. [26] reported similar decreases (19-21\%) when higher doses of salbutamol were used, whereas HELLINCKX et al. [27], and NIELSEN and BISGAARD [28] observed much smaller changes (10-13\%).

The threshold for $R 4$ to detect a significant bronchodilator response as determined in the children who never wheeze was $-43 \%$ of the baseline value. Similar cut-off values were determined for $R 6$ and $R 8$ ( $-41 \%$ and $-43 \%$, respectively; see table 2 of the online supplementary material). These cut-off values are close to previously published threshold values in healthy preschool children, which ranged from $-28 \%$ to $-42 \%$ [25-28]. On use of this threshold value, the number of responders in the groups of different wheezing phenotypes were similar (table 3). In accordance with this finding, both THAMrIN et al. [25] and HELLINCKX et al. [27] reported that use of this threshold did not allow the distinction of children with asthma or wheeze from healthy preschool children. We are aware of only one study in which preschool children at risk of persistent asthma were investigated because of repeated wheezing episodes at a young age. MAROTTA et al. [29] reported that children with suspected asthma exhibited a larger relative change in $R 5$ than children without asthma, but with a comparable baseline lung function.

When we used a cut-off value based on the absolute change to detect a positive bronchodilator response, significant differences were observed between the wheezing phenotype groups, suggesting that limits based on absolute change are more sensitive than limits based on relative change (table 3). A higher baseline airway resistance, caused by an increased bronchomotor tone, will be associated with a larger absolute response to a bronchodilator (fig. 4). Consequently, the expression of the change relative to the initial level will tend to homogenise the different responses to bronchodilation in subjects with different baseline bronchomotor tones.

TURNER et al. [5] demonstrated that a reduced pulmonary function at 1 month of age and airway responsiveness are 
independently related to persistent wheeze at 11 yrs of age. Moreover, it was recently concluded that the pattern of wheeze and the lung function level at 6 yrs of age determine the respiratory symptoms and respiratory function in adolescence [30]. The two factors that may explain the relationship between a reduced lung function at an early age and an increased wheeze during childhood are 1) wheeze due to narrow, small airways, and 2) altered airway wall properties that may result in a more pronounced wheeze. Asymptomatic infants with a history of wheeze have been reported to have a lower airway wall compliance than that of healthy age-matched controls [31]. A recent study reported that reticular basement membrane thickening is already detectable in the airways of preschool children who suffer from a severe, recurrent wheeze [32]. A diminished lung function was detected in infants 1 month of age who subsequently exhibited episodes of wheezing or coughing, suggesting that alterations in the airways may precede the onset of wheeze [33]. The findings of BRUSSEE et al. [11] and the results of our own study uniformly indicate that 4-yr-old children with persistent wheeze manifest an increased airway resistance compared with children who never wheeze. Moreover, our results on the bronchodilator response point to narrower airways being associated (at least partially) with an increased bronchomotor tone.

In summary, our study has demonstrated that children at 4 yrs of age with various wheezing phenotypes possess different baseline lung functions. Children with persistent wheeze have an elevated baseline respiratory resistance, and their bronchodilator response is more marked than that of children who never wheeze or who have early transient wheeze.

\section{SUPPORT STATEMENT}

This study was supported by grants from the Research Foundation Flanders (FWO), Belgium (number 7.0015.00) and the Flemish Government (PBO98/26/143).

\section{STATEMENT OF INTEREST}

None declared.

\section{ACKNOWLEDGEMENTS}

We are grateful to the children and parents participating in the study and to all PIPO study personnel, in particular C. Daenen, R. Vroom, D. Stappers, F. Willekens and R. Heyndrickx (all Dept of Epidemiology and Social Medicine, University of Antwerp, Antwerp, Belgium), for their contributions to this study.

\section{REFERENCES}

1 Bisgaard H, Szefler S. Prevalence of asthma-like symptoms in young children. Pediatr Pulmonol 2007; 42: 723-728.

2 Martinez FD, Wright AL, Taussig LM, et al. Asthma and wheezing in the first six years of life. N Engl J Med 1995; 332: 133-138.

3 Lombardi E, Morgan WJ, Wright AL, et al. Cold air challenge at age 6 and subsequent incidence of asthma. A longitudinal study. Am J Respir Crit Care Med 1997; 156: 1863-1869.

4 Sears MR, Greene JM, Willan AR, et al. A longitudinal, populationbased, cohort study of childhood asthma followed to adulthood. $N$ Engl J Med 2003; 349: 1414-1422.

5 Turner SW, Palmer LJ, Rye PJ, et al. The relationship between infant airway function, childhood airway responsiveness, and asthma. Am J Respir Crit Care Med 2004; 169: 921-927.
6 Håland G, Carlsen KC, Sandvik L, et al. Reduced lung function at birth and the risk of asthma at 10 years of age. N Engl J Med 2006; 355: 1682-1689.

7 Stern DA, Morgan WJ, Wright AL, et al. Poor airway function in early infancy and lung function by age 22 years: a non-selective longitudinal cohort study. Lancet 2007; 370: 758-764.

8 Stern DA, Morgan WJ, Halonen M, et al. Wheezing and bronchial hyper-responsiveness in early childhood as predictors of newly diagnosed asthma in early adulthood: a longitudinal birth-cohort study. Lancet 2008; 372: 1058-1064.

9 Sly PD, Boner AL, Björksten B, et al. Early identification of atopy in the prediction of persistent asthma in children. Lancet 2008; 372: 1100-1106.

10 Beydon N, Davis SD, Lombardi E, et al. An official American Thoracic Society/European Respiratory Society statement: pulmonary function testing in preschool children. Am J Respir Crit Care Med 2007; 175: 1304-1345.

11 Brussee JE, Smit HA, Koopman LP, et al. Interrupter resistance and wheezing phenotypes at 4 years of age. Am J Respir Crit Care Med 2004; 169: 209-213.

12 Asher MI, Keil U, Anderson HR, et al. International Study of Asthma and Allergies in Childhood (ISAAC): rationale and methods. Eur Respir J 1995; 8: 483-491.

13 Oostveen E, MacLeod D, Lorino $\mathrm{H}$, et al. The forced oscillation technique in clinical practice: methodology, recommendations and future developments. Eur Respir J 2003; 22: 1026-1041.

14 Brand PLP, Baraldi E, Bisgaard H, et al. Definition, assessment and treatment of wheezing disorders in preschool children: an evidence-based approach. Eur Respir J 2008; 32: 1096-1110.

15 Lowe LA, Simpson A, Woodcock A, et al. Wheeze phenotypes and lung function in preschool children. Am J Respir Crit Care Med 2005; 171: 231-237.

16 Kusel MM, de Klerk NH, Kebadze T, et al. Early-life respiratory viral infections, atopic sensitization, and risk of subsequent development of persistent asthma. J Allergy Clin Immunol 2007; 199: 1105-1110.

17 Lau S, Illi S, Sommerfeld C, et al. Transient early wheeze is not associated with impaired lung function in 7-yr-old children. Eur Respir J 2003; 21: 834-841.

18 Kurukulaaratchy R, Matthews S, Arshad SH. Does environment mediate earlier onset of the persistent childhood asthma phenotype? Pediatrics 2004; 113: 345-350.

19 Goldman MD, Carter R, Klein R, et al. Within- and between-day variability of respiratory impedance, using impulse oscillometry in adolescent asthmatics. Pediatr Pulmonol 2002; 34: 312-319.

20 Delacourt C, Lorino H, Fuhrman C, et al. Comparison of the forced oscillation technique and the interrupter technique for assessing airway obstruction and its reversibility in children. Am J Respir Crit Care Med 2001; 164: 965-972.

21 Lowe L, Murray CS, Custovic A, et al. Specific airway resistance in 3-year-old children: a prospective cohort study. Lancet 2002; 359: 1904-1908.

22 Marchal F, Haouzi P, Peslin R, et al. Mechanical properties of the upper airway wall in children and their influence on respiratory impedance measurements. Pediatr Pulmonol 1992; 13: 28-33.

23 Lutchen KR, Gillis H. Relationship between heterogeneous changes in airway morphometry and lung resistance and elastance. J Appl Physiol 1997; 83: 1192-1201.

24 Kaczka DW, Ingenito EP, Suki B, et al. Partitioning airway and lung tissue resistances in humans: effects of bronchoconstriction. $J$ Appl Physiol 1997; 82: 1531-1541.

25 Thamrin C, Gangell CL, Udomittipong K, et al. Assessment of bronchodilator responsiveness in preschool children using forced oscillations. Thorax 2007; 62: 814-819.

26 Malmberg LP, Pelkonen A, Poussa T, et al. Determinants of respiratory system input impedance and bronchodilator response 
in healthy Finnish preschool children. Clin Physiol Funct Imaging 2002; 22: 64-71.

27 Hellinckx J, De Boeck K, Bande-Knops J, et al. Bronchodilator response in 3-6.5 years old healthy and stable asthmatic children. Eur Respir J 1998; 12: 438-443.

28 Nielsen KG, Bisgaard H. Discriminative capacity of bronchodilator response measured with three different lung function techniques in asthmatic and healthy children aged 2 to 5 years. Am J Respir Crit Care Med 2001; 164: 554-559.

29 Marotta A, Klinnert MD, Price MR, et al. Impulse oscillometry provides an effective measure of lung dysfunction in 4-year-old children at risk for persistent asthma. J Allergy Clin Immunol 2003; 112: 317-322.
30 Morgan W, Stern DA, Sherrill DL, et al. Outcome of asthma and wheezing in the first 6 years of life: follow-up through adolescence. Am J Respir Crit Care Med 2005; 172: 1253-1258.

31 Frey U, Makkonen K, Wellman T, et al. Alterations in airway wall properties in infants with a history of wheezing disorders. Am J Respir Crit Care Med 2000; 161: 1825-1829.

32 Saglani S, Payne DN, Zhu J, et al. Early detection of airway wall remodeling and eosinophilic inflammation in preschool wheezers. Am J Respir Crit Care Med 2007; 176: 858-864.

33 Murray CS, Pipis SD, McArdle EC, et al. Lung function at one month of age as a risk factor for infant respiratory symptoms in a high risk population. Thorax 2002; 57: 388-392. 